\title{
Unusual Presentation Of Sarcoidosis: Case Report
}

${ }^{1}$ Department of Internal Medicine, County Emergency Clinical Hospital of Constanta

${ }^{2}$ Department of Oncology, County Emergency Clinical Hospital of Constanta

${ }^{3}$ University "Ovidius" of Constanta, Faculty of Medicine

\begin{abstract}
Sarcoidosis is a multisystem granulomatous disease with polymorphic manifestations, of unknown etiology, that can affect any organ in the body, but most commonly the lungs $[1,2,3]$. Extrapulmonary involvement is common, can occur in association with or in the absence of intrathoracic disease, and all organs can be involved, but it is rare to find an isolated extrapulmonary disease (less than $10 \%$ of patients) $[3,4]$. On occasion, the presentation of sarcoidosis may be atypical.

We report a case of systemic sarcoidosis, with particular clinical data: extrathoracic onset with subdiaphragmatic lymph nodes, hepatomegaly and splenomegaly.
\end{abstract}

Keywords: sarcoidosis, hepatomegaly, splenomegaly

\section{Adelina Anton}

Blv Tomis no. 145, Department of Internal Medicine, County Emergency Clinical Hospital of Constanta, Constanta, Romania email : adelinamiu@gmail.com

\section{Case report}

A 37-years-old man, farmer by profession, was admitted to the Department of Surgery for upper abdominal pain, fatigue, weight loss, with a high suspicion of gastric cancer.

Personal history and family history were insignificant.

The onset was insidious, with two months prior this admission, with progressive weakness to which weight loss (10 kg) and nonspecific upper abdominal pain were associated later.

His physical examination revealed only hepatomegaly and splenomegaly.

Laboratory studies indicated biological inflammatory syndrome (ESR, slightly elevated fibrinogen), hypergammaglobulinemia and a negative tuberculin purified protein derivative (PPD ) skin test.

Abdominal ultrasound and CT scan documented multiple subdiaphragmatic lymph nodes (periaortic, inter-aorto-caval and iliac), with diameter from 1.75 to $2.5 \mathrm{~cm}$ (Figure 1). 


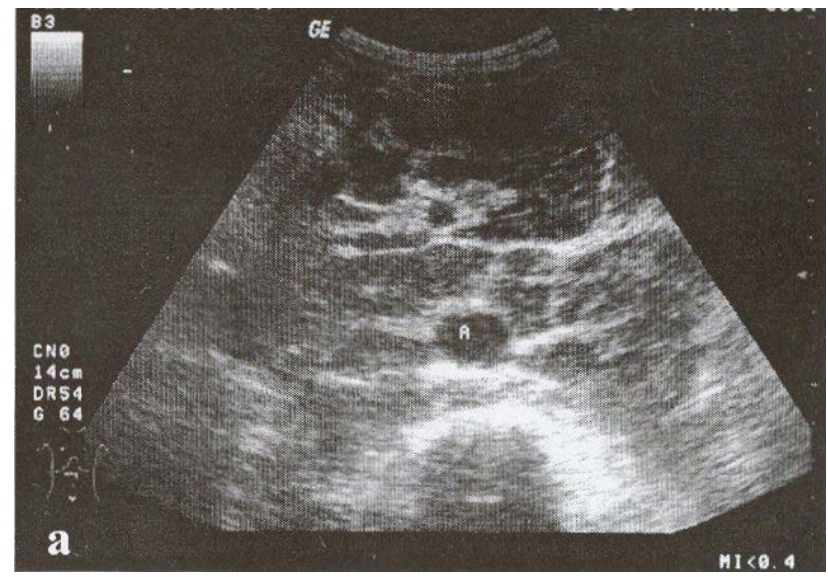

Figure. 1: Abdominal ultrasound: mutiple periaortic lymph nodes

Because the findings were highly suspicious for a digestive tumor, GI endoscopy ruled out a neoplasia with this location.

Chest radiographs, chest CT scan and pulmonary function tests were performed, but they were all normal.

At this point, the patient underwent a diagnostic laparotomy. Splenectomy, lymph node and liver biopsy nodule were performed. Pathology report showed chronic non caseating epithelioid granulomas, with giant Langhans cells and accentuated fibrosis (Figure 2).

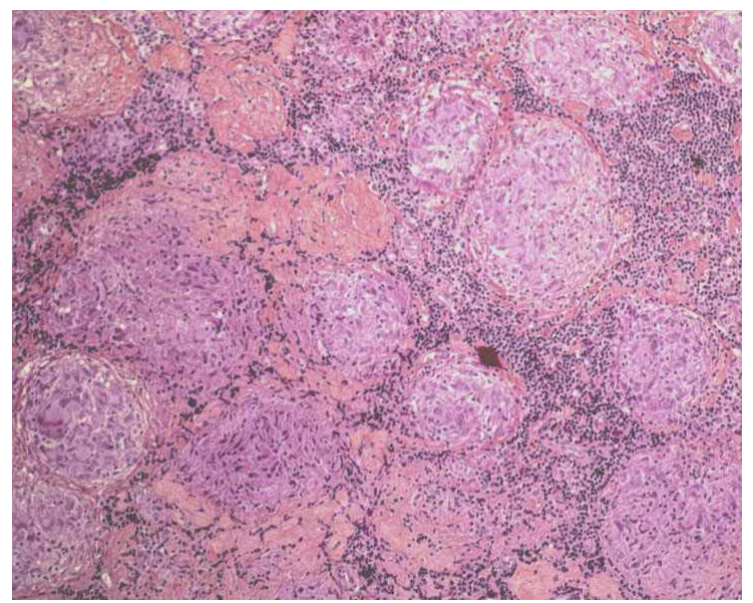

Figure. 2: Non caseating epithelioid granulomas, with giant Langhans cells and accentuated fibrosis
Positive diagnosis of extrapulmonary sarcoidosis was made.

Differential diagnosis included extrapulmonary tuberculosis, digestive tract neoplasia, malignant lymphomas, leukemias.

Steroid therapy was instituted (prednisone 1 $\mathrm{mg} / \mathrm{kg} /$ day for 30 days, then dose was gradually reduced to $10 \mathrm{mg}$ every 2 days), treatment followed for 18 months. After three months of therapy, complete regression of lymph nodes, hepatomegaly and general signs were shown . 18 months later, the treatment was discontinued due to favorable evolution, the patient following to be evaluated every 3 months, indication which he did not complied with. One year and a half after treatment discontinuation, the disease has relapsed consisting of recurrence of subdiaphragmatical lymph nodes, hepatomegaly but, this time, lung impairement was associated.

Impairement of the lungs was documented by:

1. Chest radiograph: reticulo-nodular fibrosis especially in the upper $2 / 3$ of the lung fields (Figure $3)$;

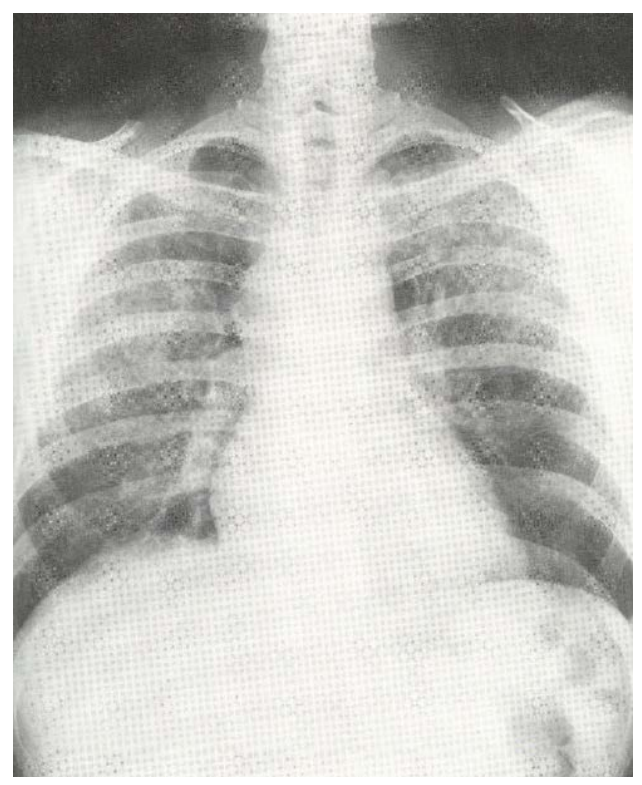

Figure 3: Chest radiography : Diffuse pulmonary fibrosis located predominantly in the upper $2 / 3$ 
2. CT pulmonary : bilateral reticular fibrosis in the upper 2/3 (Figure 4);

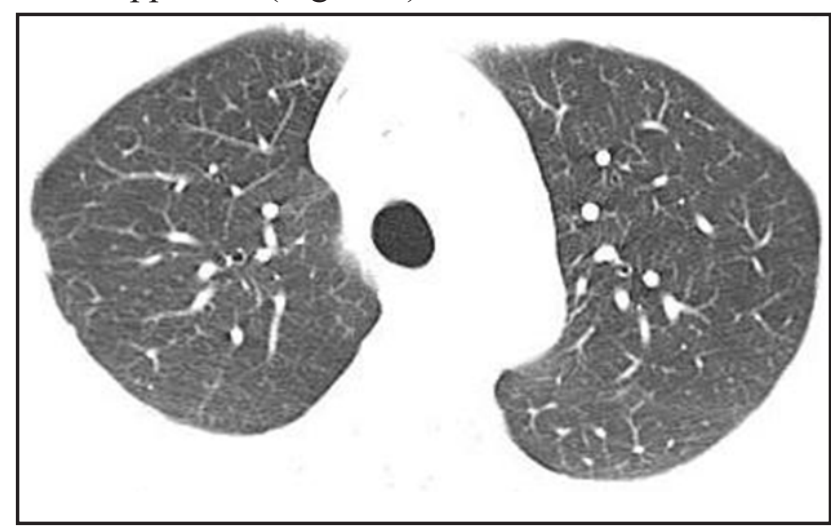

Figure. 4: CT scan showes reticular opacities with upper zone distribution

3. Pulmonary function tests: restrictive ventilatory dysfunction (FVC $=36 \%$ of predicted value, $\mathrm{FEV} 1 \%=41 \%$ of predicted value, $\mathrm{IPB}=88 \%$ ) with decreased transfer factor $(57,1 \%)$ of predicted value;

4. Bronchoscopy showed only intense hyperemia of bronchial mucosa, with intense capillary vessels (Figure 5); bronchoalveolar lavage fluid (BAL) examination: lymphocytic alveolitis (lymphocytes $46 \%$ of the total number of cells), the cytology was negative for neoplastic cells.

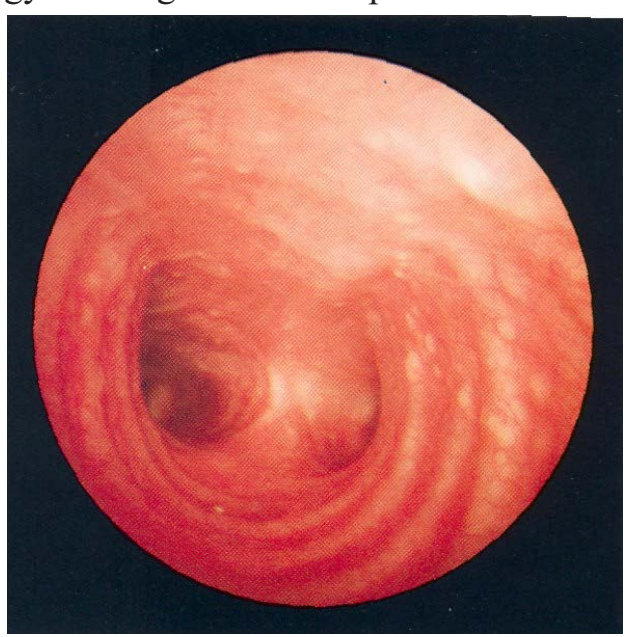

Figure 5: Bronhoscopy: intense hyperemia of bronchial mucosa, visible capillary vessels.
5. Serum angiotensin convertase (CEA) was dosed with a positive result, 33.5 U/L (N: 15-28 U/L).

Treatment with prednisone was resumed, 1 $\mathrm{mg} / \mathrm{kg} /$ day 30 days, with further reduction of the dose. After 3 months of treatment subdiaphragmatical lymph nodes disappeared and respiratory function tests were slightly improved, which allowed the progressive reduction of corticosteroid dosage to a maintenance dose of $20 \mathrm{mg}$ to 2 days.

During treatment, there was an iatrogenic complication - cortisone induced diabetes, which required hygienic-dietary measures and treatment with oral antidiabetic agents (repaglinide).

The particularity of the case is the extrathoracic onset with hepatomegaly, splenomegaly and subdiaphragmatical lymph nodes manifestations that are rare in sarcoidosis. Pulmonary impairement appeared at first relapse, after 3 years of disease evolution, and included stage 3 of sarcoidosis, with pulmonary fibrosis..

\section{Discussion}

Sarcoidosis is a granulomatous disease of unknown aetiology with various clinical presentations depending on affected organs [1,3,9]. Lung and lymphatic system are the principal localizations $[7,11,12]$. Typical clinical manifestations are easier recognized, but unsual presentations, such as nodular lesions of spleen and/or liver, are difficult to diagnose in absence of clinical suspicious of sarcoidosis $[1,3,9]$. The diagnosis of sarcoidosis could be more complicated especially if abdominal sarcoidosis represents the exclusive involvement without 
systemic disease [3,6,9]. Usually, extrapulmonary disease occurs in the context of more extensive chest disease $[13,14,15]$. Isolated splenic sarcoidosis or abdominal sarcoidosis without pulmonary or mediastinal involvement is rare, such as our patient $[4,5,13]$. We have described a patients with extrapulmonary sarcoidosis initially who later developed pulmonary involvement. Symptoms of extrapulmonary sarcoidosis are usually less characteristical for this localization. Abdominal pain and systemic symptoms, including fever, malaise and weight less occasionally occur. Laboratory tests are usually normal, but some patients with splenic involvement can develop anaemia, thrombocytopenia and neutropenia $[16,17,18]$. Our patinet had no blood counts abnormalities only positive non specific inflammatory markers and a slightly increased titer of ACE. Abdominal ultrasound scan and HRCT can easily detect the abdominal lesions. Splenomegaly (greatest splenic dimension $>14 \mathrm{~cm}$ ) is the most common splenic manifestation $[19,20]$. The organ is usually homogeneous, but multiple low-attenuating nodular lesions are occasionally noted and easily mistaken for lymphoma, metastases or infections $[13,15,19]$. Isolated or predominant hepatic nodular disease is less common than spleen impairement [2,5,13]. Besides spleen involvement, our patient presented also a hepatic nodule. In this case, for ascertain the correct diagnosis, splenectomy and hepatic nodule biopsy were required. The extent of pulmonary involvement, was evaluated both at the diagnosis and subsequent follow-up visits. At the time of diagnosis, the patient had no respiratory symptoms. We have seen that the progressive development of the disease is variable: the patient first described with initial diagnosis of extrapulmonary sarcoidosis but after that 3 years since diagnosis was made, he presented pulmonary imapirement. The treatment directed against the granulomatous inflammation is effective $[3,18]$. In this case, oral corticotherapy was administrated for 18 months, which was succesfull with complete remision of abdominal lymph adenophaties and symptoms. This made possible to discontinue the therapy and to have a follow-up check every 3 months, but the patient missed his periodic evaluation and he presented 18 months later when he developed systemic sarcoidosis. When antisarcoidosis medications are withdrawn, relapse is very common and typically occur 1 month to 1 year after therapy is tapered or discontinued $[3,18]$.

\section{Eonclusion}

Sarcoidosis is a chronic multisystem granulomatous disease. Atypical onset such as splenic or hepatosplenic involvement can occur and it is possible to be an exclusive involvement of these organs without pulmonary disease. Diagnosis of extrapulmonary sarcoidosis is difficult because , usually, is not suspected. In fact, hepatosplenic or/ and splenic determinations are often asymptomatic or accompanied by unspecific symptoms and laboratory abnormal values [3,10]. In our case, US and abdominal CT scan identified pathological changes but the diagnosis was established only with the histological examination which is required to differentiate liver and/or spleen sarcoidosis from tuberculosis, primary biliary cirrhosis, metastasis or malignant lymphoma and other granulomatous diseases $[3,10]$. After diagnosis, continual follow-up for systemic manifestations is indicated. In some cases, recurrence 
of the disease may be considered an acute exacerbation of the sarcoidosis but in our situation, the relapse may have been controlled if the patient would have been complient to follow-up visits. On one hand, this case shows that the diagnosis of sarcoidosis can be difficult when only extrapulmonary impairement is involved. Furthermore, atypical presentations may result in a delay in diagnosis as well as unnecessary treatment or diagnostic testing $[3,10]$.

\section{References}

1. Nunes, H., Bouvry, D., Soler, P. \& Valeyre, D. (2007). Sarcoidosis. Orphanet J Rare Dis. 2, 46. doi: 10.1186/1750-1172-2-46.

2. Thanos, L., Zormpala, A., Brountzos, E., Nikita, A. \& Kelekis, D. (2002). Nodular hepatic and splenic sarcoidosis in a patient with normal chest radiograph. Eur J Radiol. 41(1), 10-11.

3. Giovinale, M., Fonnesu, C., Soriano, A., Cerquaglia, C., Curigliano, V., Verrecchia, E., De Socio, G., Gasbarrini, G. \& Manna, R. (2009). Atypical sarcoidosis: case reports and review of the literature. Eur Rev Med Pharmacol Sci. 13 Suppl 1, 37-44.

4. Zia, H., Zemon, H. \& Brody, F. (2005). Laparoscopic splenectomy for isolated sarcoidosis of the spleen. J Laparoendosc Adv Surg Tech A. 15(2), 160-162. doi: 10.1089/ lap.2005.15.160

5. Judson, M.A. (2002). Hepatic, splenic, and gastrointestinal involvement with sarcoidosis. Semin Respir Crit Care Med. 23(6), 529-541. doi: $10.1055 / \mathrm{s}-2002-36517$.

6. Judson, M.A. (2007). Extrapulmonary sarcoidosis. Semin Respir Crit Care Med. 28(1), 83-101. doi: 10.1055/s-2007-970335.

7. Criado, E., Sanchez, M., Ramirez, J., Arguis, P., de Caralt, T.M., Perea, R.J. \& Xaubet, A. (2010).
Pulmonary sarcoidosis: typical and atypical manifestations at high-resolution CT with pathologic correlation. Radiographics. 30(6), 1567-1586. doi: 10.1148/rg.306105512.

8. Morgenthau, A.S. \& Teirstein, A.S. (2011). Sarcoidosis of the upper and lower airways. Expert Rev Respir Med. 5(6), 823-833. doi: 10.1586/ers.11.66.

9. Bouvry, D., Uzunhan, Y., Naccache, J.M., Nunes, H., Brillet, P.Y. \& Valeyre, D. (2008). [Atypical sarcoidosis]. Rev Med Interne. 29(1), 46-53. doi: 10.1016/j.revmed.2007.10.005

10. Facco, M., Cabrelle, A., Teramo, A., Olivieri, V., Gnoato, M., Teolato, S., Ave, E., Gattazzo, C., Fadini, G.P., Calabrese, F., Semenzato, G. \& Agostini, C. (2011). Sarcoidosis is a Th1/Th17 multisystem disorder. Thorax. 66(2), 144-150. doi: $10.1136 /$ thx.2010.140319.

11. Cox, C.E., Davis-Allen, A. \& Judson, M.A. (2005). Sarcoidosis. Med Clin North Am. 89(4), 817-828. doi: 10.1016/j.mena.2005.02.006.

12. Iannuzzi, M.C., Rybicki, B.A. \& Teirstein, A.S. (2007). Sarcoidosis. N Engl J Med. 357(21), 2153-2165. doi: 10.1056/NEJMra071714.

13. Kataoka, M., Nakata, Y., Hiramatsu, J., Okazaki, K., Fujimori, Y., Ueno, Y., Tanimoto, Y., Kanehiro, A., Tada, S. \& Harada, M. (1998). Hepatic and splenic sarcoidosis evaluated by multiple imaging modalities. Intern Med. 37(5), 449-453.

14. Zia, H., Zemon, H. \& Brody, F. (2005). Laparoscopic splenectomy for isolated sarcoidosis of the spleen. $J$ Laparoendosc Adv Surg Tech A. 15(2), 160-162. doi: 10.1089/ lap.2005.15.160.

15. Warshauer, D.M. \& Lee, J.K. (2004). Imaging manifestations of abdominal sarcoidosis. AJR Am J Roentgenol. 182(1), 15-28. doi: 10.2214/ ajr.182.1.1820015.

16. Bergoin, C., Lamblin, C. \& Wallaert, B. (2001). [Biological manifestations of sarcoidosis]. Ann Med Interne (Paris). 152(1), 34-38.

17. Baudin, B. (2005). [Angiotensin I-converting enzyme (ACE) for sarcoidosis diagnosis]. Pathol Biol (Paris). 53(3), 183-188. doi: 10.1016/j. patbio.2004.09.003

18. Judson, M.A. (2008). Sarcoidosis: clinical 
presentation, diagnosis, and approach to treatment. Am J Med Sci. 335(1), 26-33. doi: 10.1097/MAJ.0b013e31815d8276

19. Skodric-Trifunovic, V., Vucinic, V., Colovic, R., Videnovic, J., Zugic, V. \& Stojsic, J. (2004). [Liver and splenic sarcoidosis: diagnostic procedures]. Med Pregl. 57(9-10), 462-466.

20. Mohan, A., Sood, R., Shariff, N., Gulati, M.S., Gupta, S.D. \& Dutta, A.K. (2004). Sarcoidosis manifesting as massive splenomegaly: a rare occurrence. Am J Med Sci. 328(3), 170-172. 